R. D'ACHIARDI

\title{
Síndrome de anemia cardiorrenal Estudio de casos y controles anidado en una cohorte
}

\author{
Cardiorenal anemia syndrome \\ Cohort nested case-control study
}

La enfermedad renal crónica (ERC) y la anemia son patologías coexistentes, complicaciones frecuentes y factores importantes de la progresión de la insuficiencia cardiaca (IC), por lo cual Silverberg (1) en 2006 denominó esta asociación síndrome de anemia cardiorrenal: CRAS (por su siglas en inglés: Cardio Renal Anemia Syndrome).

Según el Primer Consenso Colombiano de CRAS en 2007 (2), se estableció el diagnóstico con base en los siguientes criterios:

1. IC: diagnosticada según los criterios de la Sociedad Europea de Cardiología, con signos y síntomas de IC y evidencia de disfunción sistólica o diastólica, de preferencia por ecocardiograma.

2. ERC: determinada por filtración glomerular (FG) $<60 \mathrm{cc} / \mathrm{min} . / 1.73 \mathrm{~m}^{2}$

3. Anemia: con hemoglobina $(\mathrm{Hg})<12 \mathrm{~g} / \mathrm{dL}$ en mujeres $\mathrm{y}<13 \mathrm{~g} / \mathrm{dL}$ en hombres

Se calcula que hay más de cinco millones de pacientes con IC en EEUU y 550000 nuevos casos por año (3), mientras en Europa 15 millones de habitantes tiene IC (4).

El CRAS se ha relacionado con aumento de la morbimortalidad en IC. La IC puede causar ERC progresiva y ambas pueden llevar a anemia, que su vez empeora la IC y la ERC (1).

Se debe establecer el diagnóstico clínico y paraclínico de la IC y de la anemia. En cuanto a la ERC, de hecho un factor de riesgo cardiovascular en la población general y más aún en aquella con IC, se debe detectar su presencia con la medición de la creatinina sérica y en el futuro de la Cistatina $\mathrm{C}$, y con el cálculo de la función renal desarrollando la fórmula de depuración de creatinina (DC) por Cockroft Gault $(\mathrm{CG})(\mathrm{DC}=140-$ edad (años) $\mathrm{x}$ peso $(\mathrm{Kg}) /$ creatinina $(\mathrm{mg} \mathrm{dL}) \times 72$, ajustada $\times 0.85$ en mujeres $/ 1.73 \mathrm{~m}^{2}$ ) (5), o de la FG por MDRD 4 (Modification Diet in Renal Disease) (6), y en el futuro posiblemente a través de la fórmula CKD EPI. Luego, si es del caso, se mide la DC en orina de 24 horas, incluida la medición de proteinuria, que en la actualidad según las Guías KDIGO (7), se considera criterio importante en el diagnóstico, manejo y pronóstico de la ERC; o bien con la FG isotópica. Se considera como punto de corte de disfunción renal crónica la $\mathrm{FG}<60 \mathrm{cc} / \mathrm{min} / 1.73 \mathrm{~m}^{2}$ (ERC estado 3) (Tabla1). Calcular y/o medir la función renal permite encontrar pacientes con ERC, que no son reconocidos solo con base en el análisis aislado de la creatinina sérica.

Hay pocos estudios de epidemiología del CRAS a nivel mundial. En Colombia Rodríguez y Gómez (9), analizaron la función renal en 557 pacientes de la clínica de falla cardiaca de la Fundación Clínica Shaio en Bogotá. Con DC calculada por CG, encontraron que 62.4 de los pacientes tenían DC $<60 \mathrm{cc} / \mathrm{min}$, y como factores de riesgo HTA, DM, dislipidemia y tabaquismo. No evaluaron la anemia.

Ramírez, Saldarriaga y cols., publican en este número de Acta Médica Colombiana (10) el estudio síndrome de anemia cardiorrenal, un análisis retrospectivo de 215 pacientes con IC de la Clínica Cardiovascular Santa María de Medellín. Encontraron prevalencia de CRAS de $23.2 \%$, y como otros hallazgos de importancia: fracción de expulsión promedio de 23.36, Hg promedio de 13.34 g/dL. y DC de $55.9 \mathrm{cc} / \mathrm{min}$. En cuanto a los factores de riesgo HTA en $78 \%$ y DM en $33 \%$. En lo que respecta a medicamentos recibían en orden decreciente espironolactona, inhibidores de enzima convertidora, diuréticos, digital ó antagonistas de los receptores de angiotensina II.

El registro ADHERE (Registro Americano de Insuficiencia Cardiaca Descompensada) (11) mostró alta prevalencia de disfunción renal entre los pacientes hospitalizados por IC aguda descompensada, que incrementó la mortalidad de $1,9 \%$ en quienes no la tenían hasta $7.6 \%$ en aquellos con ERC severa.

Por otra parte, la anemia es un factor independiente de progresión de la ERC y predictor de mal pronóstico en los pacientes con IC (12).
Ver artículo: página 7

Dr. Roberto D`Achiardi Rey: MD, FACP. Internista, Nefrólogo Fundación Clínica Shaio y Fundación Cardioinfantil. Nefrólogo Unidad Renal RTS Cardioinfantil, Profesor Titular de Medicina Universidad Javeriana. Profesor Clínico Principal Universidad de la Sabana.

E-mail: rodachi@gmail.com 
Tabla 1. Clasificación de la enfermedad renal crónica.

\begin{tabular}{|l|l|c|}
\hline Grado ERC & Categoría & FG cc/min/1.73 $\mathbf{~ m}^{2}$ \\
\hline G1 & Normal o alto & $\geq 90$ \\
G2 & Disminución leve & $60-89$ \\
G3a & Disminución leve a moderada & $45-59$ \\
G3b & Disminución moderada a severa & $30-44$ \\
G4 & Disminución severa & $15-29$ \\
G5 & Falla renal & $<15$ \\
\hline \multicolumn{2}{|l}{ Modificado de Guías KDIGO 2012 Clinical Practice Guideline for evaluation } \\
and management of Chronic Kidney Disease http:// www.kidney International. \\
\hline \multicolumn{2}{|l}{ Org. Kidney Int. sup 2013; 3: 91-111. } \\
\hline
\end{tabular}

Scrutinio (13) encontró prevalencia de CRAS de $21.1 \%$ en 951 pacientes con IC crónica y disfunción sistólica en Italia. Mayor edad, menor índice de masa corporal, DM, etiología Isquémica, fracción de eyección ventricular reducida y quienes no recibían tratamiento con inhibidores del sistema renina angiotensina (SRA), se relacionaron en forma independiente con el CRAS (13). El punto final primario de mortalidad por todas las causas y trasplante renal de urgencia en 3.7 años, se presentó en 404 pacientes (42.5\%), y aquellos con CRAS tuvieron mayor riesgo de presentarlo. Con base en lo anterior concluyó que la IC, la ERC y la anemia son una combinación fatal, y que a pesar de su baja prevalencia, el CRAS aumenta la mortalidad en IC crónica (13).

Cada uno de los componentes del CRAS: anemia, ERC e IC, son causa de amplia morbi mortalidad en la población general, y si lo consideramos como CRAS, se potencian y causan mayor morbi mortalidad, que como patologías individuales.

En cuanto a la terapia del CRAS, hay tres campos de acción diferentes, que siempre deben estar integrados: tratar la IC y la ERC según los criterios existentes, incluida la nefroprotección con inhibidores del SRA (en general no se recomienda darlos en combinación), y tratar la anemia con hierro oral o parenteral y con eritropoyetina u otros agentes estimulantes de la eritropoyesis. Se deben tratar siempre factores de riesgo como HTA, dislipidemia y DM, entre otros.

En conclusión, esta publicación mostró por primera vez en Colombia evidencia epidemiológica del CRAS, con prevalencia del $23.2 \%$, (10), similar a la informada por otro estudio (13). Lo anterior nos afianza en la recomendación de conocer si el paciente con IC también tiene CRAS, fácil de establecer con el análisis de $\mathrm{Hg}$, creatinina y función renal calculada, lo cual nos permite replantear los esquemas de tratamiento, dar manejo adecuado a las tres patologías, ofrecer protección de órganos blanco, prescribir ciertos medicamentos, p.e inhibidores del SRA según el caso y vigilar su administración, en cuanto con niveles reducidos de función renal, pueden causar hiperkalemia o deterioro de la función renal y finalmente, considerar que el paciente sea visto en programas de promoción y prevención. Por demás, siendo la hipertensión arterial causa frecuente de IC, y junto con la DM las dos primeras causas de diálisis crónica, y a su vez patologías de amplia prevalencia en Colombia y el mundo, deben ser estudiadas y tratadas.

Se deben realizar estudios prospectivos que nos permitan analizar la epidemiología del CRAS en Colombia y evaluar los resultados obtenidos con su manejo.

\section{Referencias}

1. Silverberg DS, Wexler D, Laina A, et al. Anemia, chronic renal disease and congestive heart failure. The cardio renal anemia Syndrome: the need for cooperation between cardiologists and nephrologists. Int Urol Nephrol 2006; 38 (2):295-310.

2. Gómez E, Bohórquez R, Comin-Coler J, D’Achiardi R, et al. CRAS - Síndrome de Anemia Cardio- Renal, I Consenso Colombiano 2007, pp 1-30 .

3. Hunt SA, Antman EM, Smith SC. AAC/AHA 2005 Guidelines Update for the Diagnosis and Management of Chronic Heart Failure in the Adult. ACC/AHA Guidelines. Circulation 2005; e154-235

4. Nieminen MS. Bohm M, et al. Guías de Práctica Clínica sobre el diagnóstico y tratamiento de la insuficiencia cardiaca aguda. Rev Esp Cardiol 2005; 58 (4): 389-429.

5. Cockcroft DW, Gault MH.Prediction of creatinine clearance from serum creatinine. Nephron 1976; 16(1): 131-141.

6. Levey AS, Bosch JP, Lewis JB, et al. A more Accurate method to estimate glomerular filtration rate from serum creatinine : a new prediction equation. Modification of Diet in Renal Disease Study Group. Ann Intern Med, 1999; 130 (6) 461-470

7. KDIGO 2012 Clinical Practice Guideline for evaluation and management of Chronic Kidney Disease. Kidney Int sup 2013; 3: $91-111$.

8. http:// www.kidney International. Org.

9. Rodríguez D, Gómez E. Prevalencia de insuficiencia renal en pacientes que asisten a la clínica de falla cardiaca. Rev Colomb Cardiol 2011; 18:144-153.

10. Ramírez JD, Saldarriaga C, Cardona MC, et al. Síndrome de anemia cardiorenal: estudio de casos y controles anidado en una cohorte. Acta Med Colomb 2013; 38: 7-11.

11. Heywood T, Fonarow G, Costanzo M, et al. High prevalence of renal dysfunction and its impact on outcome in 118.465 patients hospitalized with acute descompensated heart failure: a report from de ADHERE database. J Cardiac Fail 2007; 13 (6) 422-30

12. Palazzuoli A, Gallotta M, Lovine F, et al. Anemia in heart failure: a common interaction with renal insufficiency called the cardio-renal anemia syndrome. Int J Clin Pract 2008; 62: 281-286.

13. Scrutinio D, Passantino A, Santoro D, Catanzaro R. The cardiorenal anaemia syndrome in systolic heart failure: prevalence, clinical correlates, and long-term survival. Eur J Heart Fail 2011; 13(1):61- 67 


\section{J. Bernal, I. BRICEÑO}

\section{Estudios de HLA en Colombia}

\section{HLA research in Colombia}

Más de cincuenta años de historia de la medicina y las ciencias biológicas han sido testigos del desarrollo de los conceptos genéticos centrales a la respuesta inmune animal y humana. Los trabajos de George Snell y Jean Dausset, entre otros, identificaron los grupos de genes responsables del reconocimiento inmunológico de lo propio y lo ajeno, en el humano (sistema HLA) y en el ratón (sistema H2). De entonces a hoy se ha descrito la enorme diversidad de alelos y antígenos de histocompatibilidad, usando primero técnicas serológicas y mas recientemente describiendo la diversidad de esos sistemas en términos moleculares.

En Colombia iniciamos hace años el estudio de la variabilidad humana, utilizando tanto estos marcadores como otra serie de proteínas séricas y eritrocitarias. En 1989 se realizó un estudio de proteínas séricas (C3, BF, HP and TF) en varias poblaciones colombianas, donde se encontró una gran variación en las frecuencias génicas (Bernal y cols 1986). Posteriormente se realizaron estudios poblacionales de HLA (Bernal JE. 1991, Bernal 1995, Briceño I. 1996, Middleton 2000, Trachtenberg EA, 1996), en los que se describió la diversidad y la marcada restricción alélica en poblaciones amerindias en comparación con otros grupos poblacionales.

Igualmente, en la región del cromosoma 6 donde se encuentran los genes que codifican el HLA, se encuentran también los genes de algunos factores del complemento sérico. Tal vez, una de las primeras asociaciones claras de patología humana con este sistema, se dio con la publicación de uno de nosotros asociando los alelos del factor B del complemento sérico a la diabetes mellitus del adulto (Bernal y cols, 1979). Dausset había sospechado la asociación de algunas enfermedades al sistema HLA, particularmente de tipo autoinmune y la peculiar y persistente asociación del HLA-B27 a la espondilitis anquilosante, por causas que no se analizaran en este escrito.

En el país se han llevado a cabo varios estudios en búsqueda de asociaciones entre el HLA y algunas enfermedades. Comenzando 1990 tratamos de entender la génesis del prurigo actínico en los indígenas Chimila, encontrando primero una asociación al HLAB40 y posteriormente una al HLA-Cw4 (Bernal JE y cols, 1988, 1990) y, sin ánimo de ser exhaustivos en esta corta nota editorial, Anaya y sus colaboradores han llevado a cabo serios estudios de asociaciones a las enfermedades de tipo autoinmune (Rojas Villarraga 2010, Cruz-Tapias, 2012). Dada la importancia de este sistema en la definición de la etiología de algunas enfermedades y su papel en la medicina de trasplantes, hay aun espacio para continuar describiendo su diversidad en nuestro país, papel que cumple el artículo de Arrunategui y cols que se publica en este numero de Acta Médica Colombiana.

\section{Bibliografía}

- Arrunategui AM, Villegas A, Ocampo LA. Frecuencias alélicas, genotípicas y haplotípicas del sistema HLA clase I y II en donantes en una población del suroccidente colombiano. Acta Med Colomb 2013; 38: 16-21.

- Bernal JE, de Brigard D, Duran C, Narvaez G, Papiha SS. (1991). HLA (A, B, C and D) antigens in the Awa-Kwaiker Indians of Colombia. Tissue Antigens, 37(3), 141-4.

- Bernal JE, Duran C, Briceno I, Ortega J, Papiha SS. (1995). HLA antigens in five Amerindian groups (Yuko, Bari, Tunebo, Guane and Paez) of Colombia: results of 'Expedición Humana'. Hum Hered, 45(4), 186-91.

- Bernal JE, Duran de Rueda MM, Ordonez CP, Duran C, de Brigard D. (1990). Actinic prurigo among the Chimila Indians in Colombia: HLA studies. J Am Acad Dermatol, 22(6 Pt 1), 1049-51.

- Bernal JE, Ellis DA, Haigh J. (1979). Bf in insulin-dependent diabetes mellitus. Lancet, 2(8149), 961.

- Bernal JE, Sarmiento P, Briceno I, Papiha SS. (1989). Polymorphism of serum proteins (C3, BF, HP and TF) of six populations in Colombia. Hum Hered, 39(2), 94-8.

- Briceno I, Bernal JE, Duran C, Ortega J, Umana A, Gomez A, Papiha SS. (1996). HLA antigens in Ameridian groups of two different linguistic families from Colombia. Eur J Immunogenet, 23(1), Feb;23(1):21-8.

- Briceno I, Gomez A, Bernal JE, Papiha SS. (1996 ). HLADPB1 polymorphism in seven South American Indian tribes in Colombia. Eur J Immunogenet., 23(3), 235-40.

- Cruz-Taías, P, Pérez-Fernández OM, Rojas-Villarraga A, Rodríguez-Rodríguez A, Arango MT, Anaya JM. (2012). Shared HLA class IIin six autoimmune diseases in Latin America: a MetaAnalysis. Autoimmune Dis 2012:569728. doi: 10.1155/2012/569728. Epub 2012 Apr 19

- Middleton D, W. F. (2000). Analysis of the distribution of HLA-A alleles in populations from five continents. Hum Immunol, 61(10), 1048-52.
Ver artículo: página 16

Dr. Jaime Bernal Villegas: PhD. Director Instituto de Genética Humana, Facultad de Medicina, Pontificia Universidad Javeriana Bogotá, D.C.; Dr. Ignacio Briceño Balcázar: PhD. Instituto de Genética Humana, Profesor Titular Facultad de Medicina, Pontificia Universidad Javeriana. Bogotá, D.C. (Colombia). E-mail: jebernal@gmail.com, ignaciobricenob@hotmail.com 
- Rojas-Villarraga A, B.-C. D. (2010). HLA-Class II in Latin American patients with type 1 diabetes. Autoimmun Rev. doi: 10.1016/j.autrev.2010.05.016. Epub 2010 May 23. Review, $9(10), 666-73$.
- Trachtenberg EA, Trachtenberg EA, Keyeux G, Bernal JE, Rhodas MC, Erlich H. (1996 ). Results of Expedicion Humana. I. Analysis of HLA class II (DRB1-DQA1-DPB1) alleles and DR-DQ haplotypes in nine Amerindian populations from Colombia. Tissue Antigens, 48(3), 174-81. 